\title{
Influence of local treatments of convection upon solar $p$ mode excitation rates
}

\author{
R. Samadi ${ }^{1,2}$, F. Kupka ${ }^{3}$, M. J. Goupil ${ }^{1}$, Y. Lebreton ${ }^{4}$, and C. van't Veer-Menneret ${ }^{4}$
}

\author{
1 Observatoire de Paris, LESIA, CNRS UMR 8109, 92195 Meudon, France \\ e-mail: Reza.Samadi@obspm. fr \\ 2 Observatório Astronómico UC, Coimbra, Portugal \\ 3 Max-Planck-Institute for Astrophysics, Karl-Schwarzschild Str. 1, 85741 Garching \\ ${ }^{4}$ Observatoire de Paris, GEPI, CNRS UMR 8111, 92195 Meudon, France
}

Received 18 February 2005 / Accepted 3 July 2005

\section{ABSTRACT}

We compute the rates $P$ at which acoustic energy is injected into the solar radial $p$ modes for several solar models. The solar models are computed with two different local treatments of convection: the classical mixing-length theory (MLT) and the formulation by Canuto et al. (1996, ApJ, 473, 550, CGM). Among the models investigated here, our best models reproduce both (i) the solar radius and the solar luminosity at solar age and (ii) the observed Balmer line profiles. For the MLT treatment, the rates $P$ do significantly depend on the properties of the atmosphere, whereas for the CGM treatment, the dependence of $P$ on the properties of the atmosphere is found to be smaller than the error bars attached to the seismic measurements. The excitation rates $P$ for modes associated with the MLT models are significantly underestimated compared with the solar seismic constraints. The CGM models yield values for $P$ closer to the seismic data than do the MLT models. We conclude that the solar p-mode excitation rates provide valuable constraints and, according to the present investigation, clearly favor the CGM treatment with respect to the MLT, although neither of them yields values of $P$ as close to the observations as recently found for 3D numerical simulations.

Key words. convection - turbulence - Sun: atmosphere - stars: atmospheres - Sun: oscillations - radiative transfer

\section{Introduction}

In the outermost part of the convective zone (CZ) of intermediate mass stars, convection is highly superadiabatic because of the rapid radiative heat gains and losses of the convective fluid. In that region, entropy fluctuations are the largest, and the resulting decrease in the convective transport efficiency is compensated for by a large increase in the eddy motions, which is responsible for the oscillation mode driving. Modelling inefficient convection is complex. 3D numerical simulations are now being performed but remain still very time-consuming. Hence for massive stellar computations, 1D stellar models are used in which only simple prescriptions of convection are implemented.

Among these simplified treatments, the Canuto \& Mazzitelli (1991, CM91 hereafter) approach differs from the classic mixing length approach (MLT hereafter) in that it takes the contribution of eddies with different sizes into account in the calculation of the convective flux and velocity, while keeping the computational expenses as low as the MLT. An improved version was proposed by Canuto et al. (1996, CGM hereafter), which takes into account the feedback of the turbulence on the energy input from the source which generates turbulent convection. These multi-eddy convection models are usually refered to as Full Spectrum of Turbulence (FST) models.

Several non-local formulations of convection have also been proposed (Gough 1977; Xiong 1978, 1985; Canuto 1992, 1993; Canuto \& Dubovikov 1998). However, we focus here on the effects of proposed improvements in the description of the energy spectrum and therefore consider only local treatments and compare FST models with MLT ones.

Any model of convection must satisfy several observational constraints provided by our Sun: the solar radius at the solar age, the Balmer line profiles, and the uvby color indices. The MLT, CM91's, and CGM's local treatments have been confronted to these observational constraints (e.g. Fuhrmann et al. 1993, 1994; van't Veer-Menneret \& Megessier 1996; Smalley \& Kupka 1997; Bernkopf 1998; Heiter et al. 2002; Montalbán et al. 2004). One main result is that these observational quantities are more sensitive to the adopted value of the convective scale length of the eddies than to the formulation of convection.

Solar seismic observations provide strong additional constraints. Comparisons of theoretical oscillation frequencies with observed solar ones have shown for instance that significant improvement in the agreement between observation and model at high frequency and degree $\ell$ can be achieved with 3D simulations (Rosenthal et al. 1999). We are interested here 
in amplitudes of oscillation that can also bring several constraints on the convective process in the outer solar envelope. Indeed, the amplitudes of solar-like oscillations result from a balance between excitation and damping. Measurements of the oscillation mode growth rates (through their line-widths) and of the mode amplitudes enable the evaluation of the excitation rates $P$. Excitation of solar-like oscillations is known to be both due to turbulent convective motions through the driving by the turbulent Reynolds stresses (see Goldreich \& Keeley 1977; Balmforth 1992; Samadi \& Goupil 2001) and due to the advection of turbulent entropy fluctuations by the turbulent movements (see Samadi \& Goupil 2001). The excitation rates $P$ are thus directly related to the velocity of the convective elements and to the amount of thermal energy advected by convective motions (i.e. the convective flux). The excitation rates then depend crucially on the way the convective velocity and flux are modelled (see Houdek et al. 1999). Solar seismic measurements therefore provide - through a model of mode excitation - additional constraints on the stellar convective properties. In this framework, the goal of the present paper is to investigate the influence of different local treatments of convection on the calculation of the rates at which energy is injected into the solar radial $p$ modes and to compare our results with the solar seismic constraints.

For this purpose we compute two calibrated solar models with the Böhm-Vitense formulation of the MLT (Böhm-Vitense 1958, hereafter BV) and with the CGM multi-eddy convection treatment. In each case, the same convection formulation is adopted for the interior and the model atmosphere. Models for the internal structure are built so as to reproduce the solar radius and the solar luminosity at the solar age. The atmosphere of each model is constructed using a $T(\tau)$ law which is derived from a Kurucz's model atmosphere (Kurucz 1993) computed with the same convection formulation (as described in Heiter et al. 2002). These model atmospheres are built in order to provide the best agreement between synthetic and observed Balmer line profiles (as in van't Veer-Menneret \& Megessier 1996, for the MLT treatment) (Sect. 2). The matching of the model atmosphere with the interior model is performed - in the manner of Morel et al. (1994) - by ensuring the continuity of the temperature gradient, $\nabla$, and of the convective flux in a transition region between the interior and the atmosphere.

We also compute two models with an Eddington gray atmosphere, one with the MLT treatment and the second with the CGM formulation. These two additional models are considered for comparison purpose only. Indeed, they have an atmosphere with the same mixing-length parameter as in the interior and do not reproduce the Balmer line profiles. As a consequence, in contrast with the interior models including a Kurucz's atmosphere as described above, their atmospheres do not fulfill constraints on the properties of the convection at the surface.

Calculation of the excitation rates requires the computation of the convective flux, $F_{\mathrm{c}}$, and of the convective velocity, $v$. This is done in Sect. 3 by paying special attention to the problem of the transition region. Indeed, the continuity of $\nabla$ and of $F_{\mathrm{c}}$ through the transition region imposes a spatial variation of the mixing-length parameter in the transition region. This variable mixing-length parameter is then used in Sect. 3 to compute $v$.
Note that our approach, which is used here to compute $v$ and $P$, is different from that of Schlattl et al. (1997), who built stellar models that assume a spatially varying mixing-length parameter, with a spatial variation imposed a priori from a comparison to 2D numerical simulations of convection, in order to compute $p$ mode frequencies.

As a last step (Sect. 4), we compute the adiabatic eigenmodes and the excitation rates $P$ for each model. The adopted model of excitation is that of Samadi \& Goupil (2001, Paper I hereafter) in which the characteristic wavenumber $k_{0}$, the wavenumber dependency of the turbulent spectra, as well as the frequency component $\left(\chi_{k}\right)$ of the correlation product of the turbulent velocity field are constrained with a $3 \mathrm{D}$ simulation of the Sun as in Samadi et al. (2003c, Paper II hereafter) and Samadi et al. (2003b, Paper III hereafter). Comparison with solar seismic constraints then allows us to conclude about the best local treatment of convection in the solar case (Sect. 5).

\section{Solar models}

All solar models discussed here are computed with the CESAM code (Morel 1997) including the following input physics and numerical features:

1. Equation of state (EOS): CEFF EOS (ChristensenDalsgaard \& Däppen 1992).

2. Opacities: OPAL (Iglesias \& Rogers 1996) data, complemented by Alexander \& Ferguson (1994) data for $T \lesssim 10^{4} \mathrm{~K}$, both sets of data being given for Grevesse \& Noels (1993) solar mixture.

3. Thermonuclear reaction rates: Caughlan \& Fowler (1988).

4. Convection: either MLT or CGM's formalism. The same convection formalism has been used in the interior and in the model atmosphere.

5. Microscopic diffusion: all models include microscopic diffusion of helium and heavy elements calculated according to the simplified formalism of Michaud \& Proffitt (1993), where heavy elements are treated as trace elements.

6. Chemical composition and mixing length parameter for convection: the Grevesse \& Noels (1993) heavy elements solar mixture has been adopted. The constraint that solar models have the observed solar luminosity and radius at solar age yields the initial helium content $Y_{0}$ and the mixing length parameter of the interior model $\alpha_{\mathrm{i}}$ (solar model calibration). Microscopic diffusion modifies the surface composition, therefore the initial ratio of heavy elements to hydrogen $(Z / X)_{0}$ is adjusted so as to get the ratio $(Z / X)_{\odot}=0.0245$ at solar age.

7. The models were calculated with 285 shells in the atmosphere and about 2000 shells in the interior.

The CGM formulation of convection is implemented according to Heiter et al. (2002). In contrast with Heiter et al. (2002), we use a characteristic scale length of convection for the two formulations which is the mixing-length $\Lambda=\alpha H_{p}$, where $H_{p}$ is the pressure scale heigth and $\alpha$ the mixing-length parameter, which can be different in the interior and in the atmosphere. 


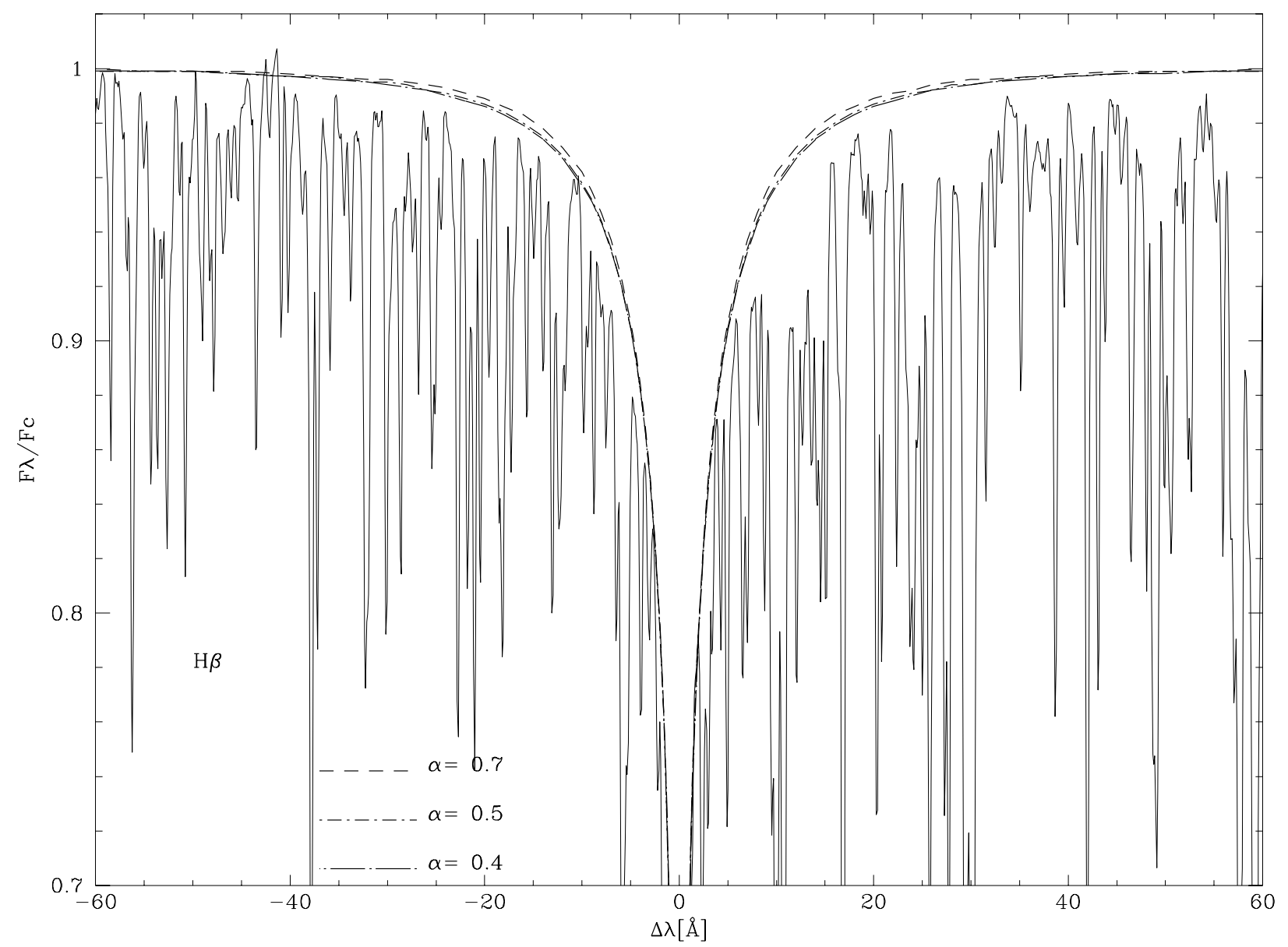

Fig. 1. The observed solar $\mathrm{H}_{\beta}$ profile is compared to theoretical ones computed with CGM models and assuming different values for $\alpha_{\mathrm{a}}$. Abscissae are distances in $\AA$ from the line center, and ordinates are the flux in the profile normalized to the continuum. The large scatter is due to the presence of many spectral lines which overlap and cause an apparent enlargement of the true profile.

\subsection{The "Kurucz models" (KMLT and KCGM models)}

We consider here two stellar models: one computed with the MLT formulation of convection and the second one with the CGM formulation. They will be labelled hereafter as KMLT model and KCGM model, respectively.

Treatment of the atmosphere: the model atmospheres of those models are computed using the ATLAS 9 code (Kurucz 1993) as described in Heiter et al. (2002). Solar model atmospheres are built assuming different values for $\alpha_{\mathrm{a}}$, the mixinglength parameter assumed for the model atmosphere: $\alpha_{\mathrm{a}}=$ $0.4,0.5,0.6,0.7$. The model atmospheres with $\alpha_{\mathrm{a}}=0.4$ and $\alpha_{\mathrm{a}}=0.5$ provide the best agreement between synthetic and observed $\mathrm{H}_{\beta}$ Balmer line profiles for the two formulations of convection. This is shown in Fig. 1 for the CGM model atmosphere. For the MLT treatment, see Fuhrmann et al. (1993, 1994) and van't Veer-Menneret \& Megessier (1996). Above $\alpha_{\mathrm{a}} \simeq 0.6$, the synthetic profile rapidly departs from the observed one, as well as the effective temperature $T_{\text {eff }}$ from the known solar $T_{\text {eff }}$.

There are no significant differences for the $\mathrm{H}_{\beta}$ Balmer line profile between the model atmospheres with $\alpha_{\mathrm{a}}=0.4$ and $\alpha_{\mathrm{a}}=0.5$. Among those model atmospheres we adopt arbitrarily those with $\alpha_{\mathrm{a}}=0.5$. Indeed, choosing the model atmospheres with $\alpha_{\mathrm{a}}=0.4$ instead of $\alpha_{\mathrm{a}}=0.5$ will not change the conclusions of this article. For each formulation of convection we then obtain a $T(\tau)$ law.

The atmospheres of the KMLT and KCGM stellar models are recomputed according to the procedure described in Morel et al. (1994) from the $T(\tau)$-laws mentioned above; the fit between interior (where the diffusion approximation is valid) and atmosphere is performed in a region where $\tau_{1} \lesssim \tau \lesssim \tau_{2}$ (an acceptable range of values for $\tau_{1}$ and $\tau_{2}$ is discussed below). In the interior region where $\tau \gtrsim \tau_{2}$, the temperature gradient $\nabla_{\mathrm{i}}$ is obtained from the MLT or CGM formalism. In the atmospheric region, where $\tau \lesssim \tau_{1}$, the temperature gradient $\nabla_{\mathrm{a}}$ is computed using the $T-\tau$ law of the model atmosphere built with the same model of convection as in the interior. In the transition region, where $\tau_{1} \lesssim \tau \lesssim \tau_{2}$, in order to ensure the continuity of the temperature gradient, $\nabla$ is obtained by a linear interpolation of $\nabla_{i}$ and $\nabla_{\mathrm{a}}$ as a function of the optical depth as follows:

$\nabla=\beta(\tau) \nabla_{\mathrm{a}}+(1-\beta(\tau)) \nabla_{\mathrm{i}}$

where $\beta(\tau)=\left(\tau_{2}-\tau\right) /\left(\tau_{2}-\tau_{1}\right)$.

Once the temperature gradients of the interior and the atmosphere are linked together in the transition region according to Eq. (1), we compute afterward in that region the convective 
flux and an equivalent mixing-length parameter (i.e. a depthdependent mixing-length parameter) as explained in Sect. 3.

Acceptable ranges for $\tau_{1}$ and $\tau_{2}$ : using a Newton-Raphson scheme, $\nabla_{\mathrm{i}}$ is adjusted in order that $F_{\mathrm{c}}^{(\mathrm{i})}+F_{\mathrm{rad}}^{(\mathrm{i})}=L_{\odot} / 4 \pi r^{2}$, where $L_{\odot}$ is the luminosity of the Sun and $F_{\text {rad }}^{(\mathrm{i})}$ is the radiative flux of the internal model. Calculation of $F_{\mathrm{c}}^{(\mathrm{i})}$ assumes the diffusion approximation for the radiative transfer for $F_{\text {rad }}$. This approximation is valid at rather high values of $\tau$, typically $\tau \gtrsim 10$ (see Morel et al. 1994). Therefore, $\tau_{1}$ cannot be much smaller than $\tau \simeq 10$. Otherwise, $\nabla_{\mathrm{i}}$ will have an unrealistic contribution to $\nabla$ below $\tau \simeq 10$. On the other hand, for the calculation of $F_{\mathrm{c}}^{(\mathrm{a})}$, the radiative flux $F_{\mathrm{rad}}$ is based on a Kurucz's model atmosphere, which treats the radiative transfer more realistically than the diffusion approximation.

The Kurucz model atmosphere is based on the Kurucz $(1992,1993)$ opacity tables which are given up to $T \simeq 2 \times 10^{5} \mathrm{~K}$

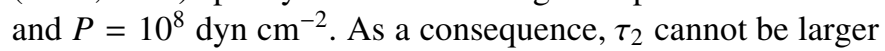
than $\tau \simeq 10^{7.5}$, i.e. layers for which $T \simeq 30000 \mathrm{~K}$. In order to ensure a satisfactory continuity of the temperature gradient, $\tau_{2}$ must be sufficiently larger than $\tau_{1}$. On the other hand, the transition region should be as small as possible; i.e. $\tau_{2}-\tau_{1}$ must be as small as possible. The main constraint for this region is thus to avoid discontinuities between the interior and the atmosphere. It is defined through an empirical procedure rather than based on a strict physical theory.

In practice, we find that $\tau_{1}=4$ is the minimal acceptable value for $\tau_{1}$; below this value the bias introduced by the diffusion approximation has a significant effect on $F_{\mathrm{c}}$. In addition, we find that above $\tau_{2} \simeq 50$, the convective velocity $v$ (see Sect. 3.2) shows a pronounced "kink" at $\tau=\tau_{2}$ for the CGM model (see Fig. 3, such a "kink" is also observed for $F_{\mathrm{c}}$, but it is less pronounced). On the other hand, the choice of $\tau_{2}=20$ avoids the angular point. For the MLT model, whatever the value of $\tau_{2}, v$ shows such a feature. This is a consequence of the much higher values of the mixing length parameter required in the interior in order to still obtain the correct solar radius, if a lower value of $\alpha$ is also used in regions further within the envelope. The requirement of matching $R_{\odot}$ hence provides a more stringent upper limit for the choice of $\tau_{2}$ (cf. also the discussion in Montalbán et al. 2004 on the computation of solar entropy as a function of radius). In the following, we will consider $\tau_{1}=4$ and $\tau_{2}=20$ as our optimal choice.

Calibration: the mixing-length parameter $\alpha_{i}$ for the internal structure, $Y_{0}$, and $(Z / X)_{0}$ are adjusted such that the stellar model simultaneously reproduces the solar radius, the solar luminosity, and the observed ratio $(Z / X)_{\odot}=0.0245$ at the solar age. The calibration yields $(Z / X)_{0}=0.0279$ and $Y_{0}=0.275$. At solar age, the helium abundance in the convection zone is $Y=0.246$, in reasonable agreement with the value $Y=0.249 \pm 0.003$ obtained from seismology (Basu 1997). Table 1 gives the calibrated values of the mixing-length parameters $\alpha_{\mathrm{i}}$ for each complete solar model. The radius resulting from the adjustement of $\alpha_{\mathrm{i}}$, as well as the size of the convective zone, are given in Table 2. All the interior models have a depth of the convective zone of $\simeq 0.286 R_{\odot}$, which is in good agreement with the value of $0.287 \pm 0.003 R_{\odot}$ determined seismically by Christensen-Dalsgaard et al. (1991).
Table 1. Values of the mixing-length parameters of the KCGM and KMLT models: $\alpha_{\mathrm{i}}$ (for the interior) and $\alpha_{\mathrm{a}}$ (for the model atmosphere). $\alpha_{\mathrm{i}}$ results from the calibration of the full model while $\alpha_{\mathrm{a}}$ is fixed (see Sect. 2.1).

\begin{tabular}{ccc}
\hline \hline model & $\alpha_{\mathrm{i}}$ & $\alpha_{\mathrm{a}}$ \\
\hline KMLT & 2.51 & 0.50 \\
KCGM & 0.78 & 0.50 \\
\hline
\end{tabular}

Table 2. $\Delta R \equiv R-R_{\odot}$, where $R_{\odot}$ is the radius at the photosphere (i.e. at $T=T_{\text {eff }}$ ), and depth of the convective zone (CZ) for the KCGM and KMLT models. These quantities are given with respect to the solar radius $R_{\odot}$ (we assume the Guenther et al. (1992) value of $R_{\odot}$ ).

\begin{tabular}{lll}
\hline \hline model & $\Delta R / R_{\odot}$ & depth CZ \\
\hline KMLT & $-10^{-6}$ & 0.2860 \\
KCGM & $510^{-6}$ & 0.2859 \\
\hline
\end{tabular}

Table 3. Values of the the mixing-length parameter $\alpha$ of the ECGM and EMLT models obtained for calibrated solar models.

\begin{tabular}{cc}
\hline \hline model & $\alpha$ \\
\hline EMLT & 1.76 \\
ECGM & 0.69 \\
\hline
\end{tabular}

\subsection{Eddington approximation based models (EMLT and ECGM models)}

For comparison purposes, we consider two additional stellar models here with an Eddington classical gray atmosphere instead of the Kurucz atmosphere models described in Sect. 2.1. One of these models assumes the MLT formulation of convection and the other the CGM formulation. In the following they will be labelled as EMLT model and ECGM model, respectively. The mixing-length parameter $\alpha$ of these models (the same $\alpha$ in the interior as in the atmosphere) is adjusted in order to reproduce the solar luminosity and radius at the solar age. However, as mentioned in the introduction, these models do not reproduce the Balmer line profiles. Table 3 gives the calibrated values of the mixing-length parameters.

\subsection{Comments}

With the CGM treatment, $\alpha_{\mathrm{i}}$ is found to be less than one and closer to $\alpha_{\mathrm{a}}=0.5$. In contrast, with the MLT treatment the value of $\alpha_{\mathrm{i}}$ is much larger than $\alpha_{\mathrm{a}}=0.5$.

The CGM models result in a much lower value for the mixing-length parameter ( $\alpha_{\mathrm{i}}$ for the KCGM model and $\alpha$ for the ECGM model) than the MLT models, because the convection in nearly adiabatic regions is more efficient with the CGM formulation than with the MLT one. Indeed, for the same value of the mixing-length parameter and in the region of high convective efficiency (below the superadiabatic region), the CGM treatment predicts a convective flux ten times larger than the MLT one for a given superadiabatic gradient. In a solar model, this behavior results in a gradient closer to the adiabatic one below a smaller superadiabatic zone in comparison to the MLT case (Canuto et al. 1996). At the top of the quasi-adiabatic 


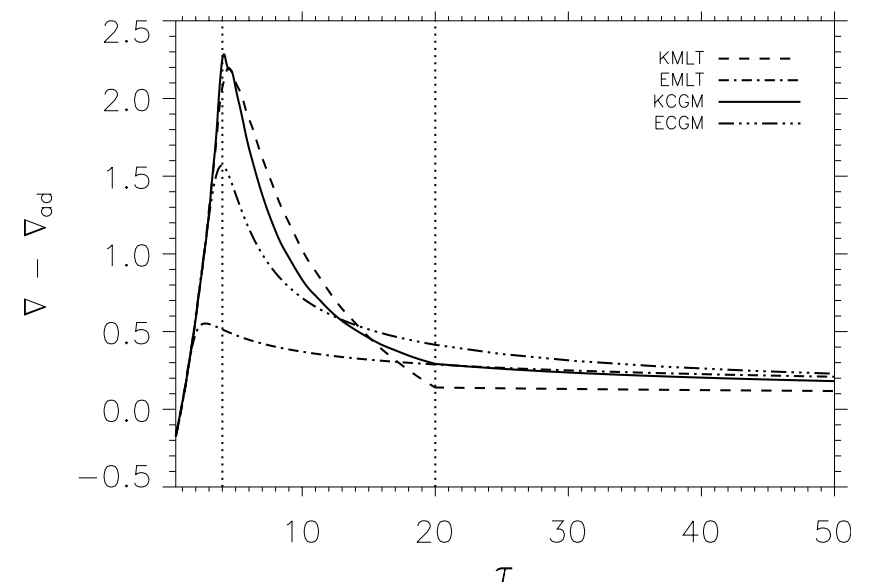

Fig. 2. The superadiabatic gradient $\nabla-\nabla_{\text {ad }}$ is plotted versus the optical depth $\tau$ in the outer region for the KMLT model (dashed line), EMLT model (dot-dashed line), ECGM model (dot-dot-dot-dashed line), and KCGM model (solid line). The dotted vertical lines correspond to the layers where $\tau=\tau_{1}=4$ and $\tau=\tau_{2}=20$, respectively, and delimit the transition region.

region, energy is predominantly transported by convection, such that $F_{\mathrm{c}} \simeq F$ where $F_{\mathrm{c}}$ is the convective flux and $F$ the total energy flux. Above, convective transport is no longer efficient $\left(F_{\mathrm{c}}<F\right)$. Therefore, the solar energy flux $F$ at the top of the quasi-adiabatic region can be reproduced for the CGM models with a lower value of the mixing-length parameter than the one required for the MLT.

The superadiabatic gradients are displayed in Fig. 2 as a function of $\tau$. The angular point observed in $\nabla-\nabla_{\text {ad }}$ at the optical depth $\tau \simeq 20$ for the KMLT model corresponds to the matching point; i.e. at $R_{\odot}-r \simeq 100 \mathrm{~km}$, where $R_{\odot}$ is the solar radius at the photosphere, defined to be where $\tau=2 / 3$ and calibrated at the precision level given in Table 2 . It results from the large difference between $\alpha_{\mathrm{i}}$ and $\alpha_{\mathrm{a}}$. This difference is much smaller for the KCGM model, and therefore the "kink" at $\tau \simeq 20$ is much less pronounced.

Our last comment concerns the large difference in the value of the mixing-length parameter between the KMLT and the EMLT models. Both models differ only by the structure of their uppermost layers located $50-100 \mathrm{~km}$ below the photosphere (see Fig. 3, middle), which represent a tiny fraction of the convection zone depth. The KMLT has a model atmosphere in which convection is much less efficient than that of the EMLT model as a consequence of the fact that $\alpha_{\mathrm{a}}=0.5$ in the Kurucz's model atmosphere (see Sect. 2.1). This is why the superadiabatic gradient, $\nabla-\nabla_{\text {ad }}$, at that depth reaches much higher values for the KMLT model than for the EMLT model. Now if $\nabla-\nabla_{\text {ad }}$ from two models are vastly different, so is the entropy jump of both. Hence, if a certain entropy of the interior convection zone and thus a certain radius of the convection zone are to be matched, a much more drastic change in $\alpha$ is required with the KMLT model to avoid to large an entropy jump (see a detailed discussion in Montalbán et al. 2004).
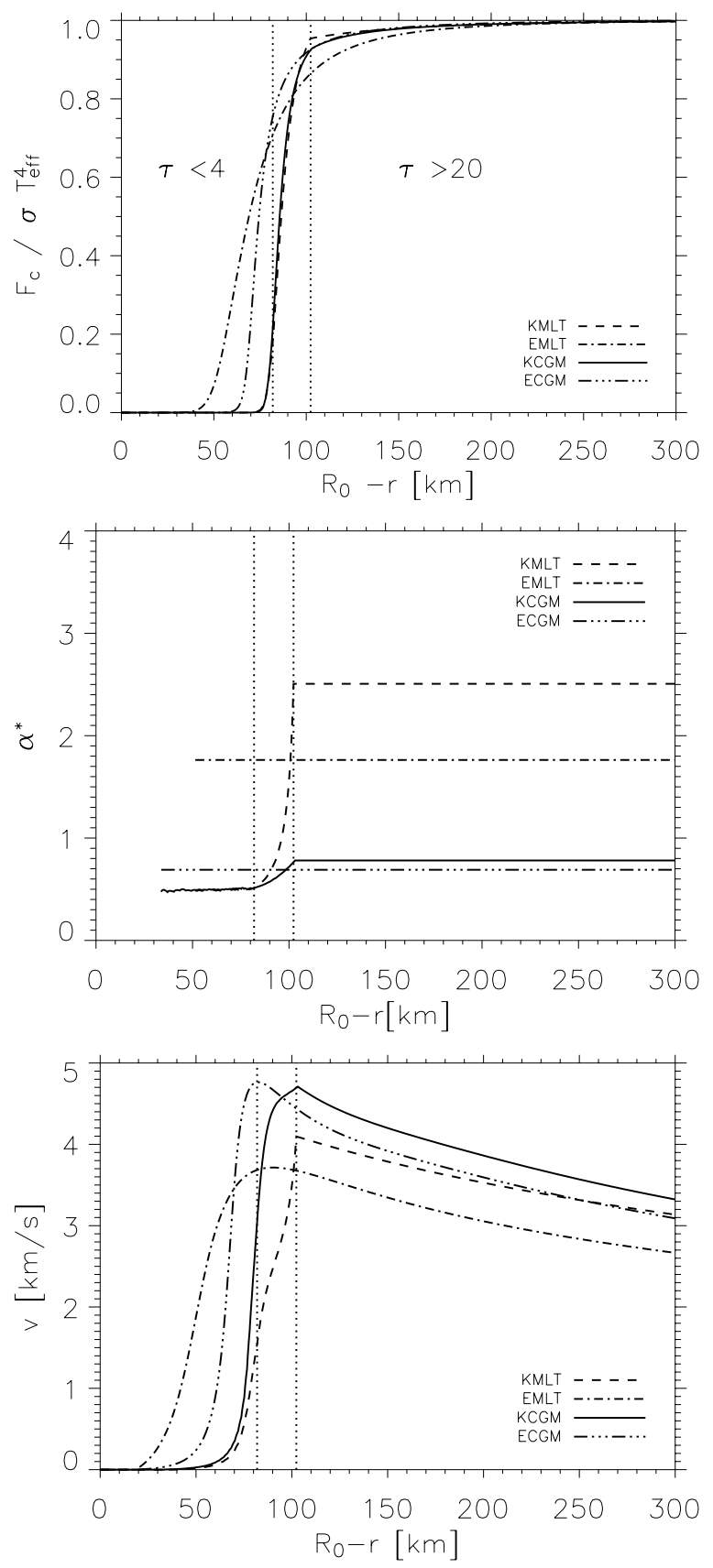

Fig. 3. Top: $F_{\mathrm{c}}$ is plotted versus $R_{\odot}-r$ for the KMLT model (dashed curve), EMLT model (dot-dashed line), ECGM model (dot-dot-dotdashed line), and KCGM model (solid line). As in Fig. 2, the dotted vertical lines delimit the transition region. Middle: same as the top panel for $\alpha^{*}$ (see Sect. 3.2). Bottom: same as the top panel for the root mean square of the convective velocity, $v$.

\section{Convective velocity and entropy fluctuations}

\subsection{Convective flux}

Part of the mode excitation rates stems from the advection of turbulent entropy fluctuations by turbulent motions (the socalled "entropy source term"). This term scales - see Paper I - as $\alpha_{s}^{2}\left\langle s_{\mathrm{t}}^{2}\right\rangle u_{0}^{2}$, where $s_{t}$ represents the entropy fluctuations due to turbulent convection, $\alpha_{s} \equiv(\partial p / \partial s)_{\rho}, \rho, s$, and $p$ are, respectively, the density, the entropy and the pressure, \langle\rangle , denotes 
spatial and time average, $u_{0}^{2} \equiv 1 / 3\left\langle\boldsymbol{u}^{2}\right\rangle$, where $\boldsymbol{u}$ is the velocity field associated with the turbulence. The factor $1 / 3$ arises from the simplifying assumption made in Paper I that the acoustic emission is injected into the p-mode isotropically in all three directions.

The "entropy source term" scales as the square of the convective flux $F_{\mathrm{c}}$. Indeed, we show (see Appendix A) that it scales as $\left(\alpha_{s} / \rho_{0} T_{0}\right)^{2}(\bar{\Phi} / 3) F_{\mathrm{c}}^{2}$, where $T_{0}$ and $\rho_{0}$ are the mean temperature and density, respectively, and $\bar{\Phi}$ is the spatially averaged anisotropy factor, which is defined as (Gough 1977):

$\bar{\Phi}(r) \equiv \frac{\left\langle u^{2}\right\rangle}{\left\langle u_{z}^{2}\right\rangle} \equiv \frac{v^{2}}{w^{2}}$

where $u_{z}$ is the vertical component of $u$, and $v$ and $w$ are defined as $v^{2} \equiv\left\langle u^{2}\right\rangle$ and $w^{2} \equiv\left\langle u_{z}^{2}\right\rangle$, respectively.

For the CGM formulation, $F_{\mathrm{c}}$ is computed according to Eqs. (2) and (17)-(21) in Heiter et al. (2002), and for the MLT treatment, it is calculated according to Eq. (14.118) in Cox (1968, Chap. 14).

$F_{\mathrm{c}}$ can be viewed as function of $\alpha$ and $\nabla, F_{\mathrm{c}}=h(\nabla, \alpha)$, where $h$ is given by the adopted model of convection.

ECGM and EMLT models: for these two models only one mixing-length parameter is involved and $F_{\mathrm{c}}$ can directly be retrieved from $\alpha$ and $\nabla$.

KCGM and KMLT models: in the outer region $\left(\tau<\tau_{1}\right)$, as well as in the interior region $\left(\tau>\tau_{2}\right)$, values of $F_{\mathrm{c}}$ can be directly retrieved from $\alpha$ and $\nabla$. In the transition region $\left(\tau_{1}<\tau<\tau_{2}\right.$ ), however, we have to deal with two functions for the convective flux: $F_{\mathrm{c}}^{(\mathrm{i})}=h\left(\nabla_{\mathrm{i}}, \alpha_{\mathrm{i}}\right)$, the convective flux calculated as in the interior, and $F_{\mathrm{c}}^{(\mathrm{a})}=h\left(\nabla_{\mathrm{a}}, \alpha_{\mathrm{a}}\right)$, the convective flux calculated for the atmosphere. As a result of Eq. (1), the convective flux $F_{\mathrm{c}}$ of the model in the transition region can be related to $F_{\mathrm{c}}^{(\mathrm{i})}$ and $F_{\mathrm{c}}^{(\mathrm{a})}$ as follows:

$F_{\mathrm{c}}=\lambda(\tau) F_{\mathrm{c}}^{(\mathrm{a})}+(1-\lambda(\tau)) F_{\mathrm{c}}^{(\mathrm{i})}$

where $\lambda(\tau)$ is - like $\beta(\tau)$ (Eq. (1)) - a function of $\tau$, which ensures the continuity of the convective flux. This function must decrease with $\tau$ and must fulfill $\lambda(\tau)=1$ for $\tau \leq \tau_{1}$ and $\lambda(\tau)=0$ for $\tau \geq \tau_{2}$. We point out that both $F_{\mathrm{c}}^{(\mathrm{i})}$ and $F_{\mathrm{c}}^{(\mathrm{a})}$ fulfill $F_{\mathrm{c}}+F_{\text {rad }}=L_{\odot} / 4 \pi r^{2}$ at any optical depth $\tau$. Just as for $\beta(\tau)$, the choice $\lambda(\tau)$ is rather arbitrary. As for the case of $\nabla$ (Eq. (1)), we assume $\lambda(\tau)=\beta(\tau)=\left(\tau_{2}-\tau\right) /\left(\tau_{2}-\tau_{1}\right)$ for $\tau_{1}<\tau<\tau_{2}$. Figure 3 shows $F_{\mathrm{c}}$ as a function of $\tau$ for the KMLT and KCGM models.

Calculation of the driving by the entropy source term requires, in addition to the convective flux $\left(F_{\mathrm{c}}\right)$, a model for the mean anisotropy $(\bar{\Phi})$. In the CGM model of convection, the expressions for $v^{2}$ and $F_{\mathrm{c}}$ do not depend explicitly on the mean anisotropy factor $\bar{\Phi}$. However, CGM adopt a model of anisotropy which fixes the ratio $x \equiv k_{h} / k_{v}$, where $k_{h}$ and $k_{v}$ are the horizontal and vertical wavenumbers associated with the eddy of wavenumber $k$ (note that $k^{2}=k_{h}^{2}+k_{v}^{2}$ ). As a result of that model, for the largest scales $x=1 / 2$, while it increases quadratically with the total wavenumber $k$ for the smaller scales. Let us define $\Phi(k) \equiv u^{2}(k) / u_{z}^{2}(k)$, a $k$-dependent anisotropy factor. For an incompressible fluid - a property assumed by the models investigated here (see also CM91) - one can show that $\Phi(k)=1+x^{-1}$. Hence $x=1 / 2$ implies $\Phi=3$, i.e. an isotropic velocity field. As a result of its functional dependence on $k$, from the larger scales $\left(k \sim k_{0}\right)$ to the smaller scales $\left(k \gg k_{0}\right) \Phi(k)$ decreases in the CGM model from $\sim 3$ towards $\sim 1$. However, we recall that the model of stochastic excitation (MSE) we consider is basically isotropic. The anisotropy is taken into account only at large scales through Gough's mean anisotropy factor $(\bar{\Phi})$. Therefore, although CGM's treatment adopts a model where the anisotropy varies with $k$, we are left with the inconsistency that the turbulent spectrum, $E(k)$, assumed for the MSE (see Sect. 4.1), is isotropic along the turbulent cascade. A possible anisotropy is only taken into account at large scales through $\bar{\Phi}$. But as the modes are predominantly excited by turbulent eddies with $k \sim k_{0}$, which carry most of the kinetic energy, this approximation is not expected to affect our prediction significantly. Hence, we assume $\bar{\Phi}=3$ for the CGM models in the discussion given below.

\subsection{Convective velocity}

Driving of the oscillation modes by the Reynolds term is proportional to $\rho_{0} v^{4}$.

Like the convective flux, the convective velocity $v$ can be viewed as a function of $\nabla$ and $\alpha$, i.e. $v=f(\nabla, \alpha)$, where the function $f$ depends on the formulation of convection. For the CGM treatment, $v=f(\nabla, \alpha)$ is computed according to Eqs. (88)-(90) of CGM. For the MLT approach, we first compute $w(\nabla, \alpha)$ according to Eq. (14.110) of Cox (1968, Chap. 14). We then deduce $v=f(\nabla, \alpha)$ from Eq. (2) with $\bar{\Phi}=2$ consistently with BV's formulation of the MLT (see Gough 1977).

ECGM and EMLT models: as for the convective flux, $v$ can be retrieved from $\alpha$ and $\nabla$.

KCGM and KMLT models: in the outer region (i.e. $\tau<\tau_{1}$ ), as well as in the inner region $\left(\tau>\tau_{2}\right)$, only one $\alpha$ and one $\nabla$ are defined. In those regions $v$ is computed as $v=f(\nabla, \alpha)$. However, in the transition region we must deal with two different values of $\alpha$ (namely $\alpha_{\mathrm{i}}$ and $\nabla_{\mathrm{i}}$ from the inner region and $\alpha_{\mathrm{a}}$ and $\nabla_{\mathrm{a}}$ from the atmosphere) and $v$ is not a linear function of $\alpha$. We thus face the difficulty of properly defining a convective velocity consistent with $F_{\mathrm{c}}$ (Eq. (3)) in this region.

We proceed as follows: $\nabla(\tau)$ and $F_{\mathrm{c}}(\tau)$ are defined by Eqs. (1) and (3), respectively. Then, at fixed $\nabla$ and $\tau$, we define an equivalent mixing-length parameter, $\alpha^{*}$, such that $F_{\mathrm{c}}=h\left(\nabla, \alpha^{*}\right)$. This parameter is variable with depth. We next compute $v=f\left(\nabla(\tau), \alpha^{*}\right)$ which is thus consistent with $F_{\mathrm{c}}$ of Eq. (3).

Figure 3 (middle and bottom) shows $\alpha^{*}$ and $v$ as a function of depth. For the MLT models, our calculation of $v$ assumes $\bar{\Phi}=2$, which is consistent with BV's formulation of the MLT (see Gough 1977), and for the CGM models it assumes $\bar{\Phi}=3$.

\subsection{Comments}

As shown in Fig. 3, the EMLT and KMLT models have very different convective structures: up until close to the top of the superadiabatic region (located at $R_{\odot}-r \sim 70 \mathrm{~km}$ for those 
models), the EMLT model results in a convective velocity and convective flux smaller than those of the KMLT model. This is a consequence of the fact that $\alpha^{\mathrm{EMLT}}<\alpha_{\mathrm{i}}^{\mathrm{KMLT}}$ (see Tables 1 and 3). On the other hand, the EMLT model results in larger $v$ and $F_{\mathrm{c}}$ above the top of the superadiabatic region because $\alpha^{\mathrm{EMLT}}>\alpha_{\mathrm{a}}^{\mathrm{KMLT}}=0.5$.

In contrast with the MLT models, the KCGM and ECGM models have rather similar convective structures. Indeed, $v^{\mathrm{KCGM}}$ and $v^{\mathrm{ECGM}}\left(F_{\mathrm{c}}^{\mathrm{KCGM}}\right.$ and $F_{\mathrm{c}}^{\mathrm{ECGM}}$ resp.) have very similar shapes. This in turn is a consequence of the fact that the CGM models require values of $\alpha_{i}^{\mathrm{KCGM}}$ and of $\alpha^{\mathrm{ECGM}}$ close to that one required for the atmosphere $\left(\alpha_{a}=0.5\right)$.

For the KMLT model, there is a pronounced "kink" at the bottom boundary of the transition region (i.e. at $\tau=20$ or $R_{\odot}-$ $r=100 \mathrm{~km}$ ), especially for $v$. For the KCGM model, the "kink" is much less important. These features are directly connected with the angular point observed for $\nabla$ in that layer (see Sect. 2.3 and Fig. 2).

At the bottom of the transition region (i.e. at $\tau=\tau_{2}$ ), for both the KMLT and KCGM models, $\alpha^{*}(\tau)$ reaches - as expected - the "interior" value of the mixing-length parameter (i.e. $\alpha_{\mathrm{i}}$ ), namely: $\alpha_{\mathrm{i}}^{\mathrm{KCGM}}=0.78$ and $\alpha_{\mathrm{i}}^{\mathrm{KMLT}}=2.51$. At the top of the transition region (i.e. at $\left.\tau=\tau_{1}\right), \alpha^{*}(\tau)$ reaches for both the KCGM and KMLT models the asymptotic value $\alpha^{*} \simeq 0.50$.

\section{Calculation of the solar $p$ mode excitation rates}

\subsection{Procedure}

We compute the rate $P$ at which acoustic energy is injected into solar radial $p$-modes according to the theoretical model of stochastic excitation of Paper I, and assume here - as in Paper II and Paper III - that injection of acoustic energy into the modes is isotropic and consider only radial $\mathrm{p}$ modes. The rate at which a given mode with frequency $\omega_{0}=2 \pi v_{0}$ is excited is then calculated with the set of Eqs. (1)-(11) of Paper III and Eq. (3) of Samadi et al. (2005).

The calculation requires the knowledge of three different types of quantities. First of all, quantities which are related to the average properties of the medium:

- the mean density $\rho, \alpha_{s}$ (Eq. (A.5)), the mean square convective velocity $v^{2}$, and the mean square of the entropy fluctuations $s^{2}$ (Eq. (A.6)). They are obtained from the equilibrium models as explained in Sect. 3.

Secondly, quantities which are related to the oscillation modes:

- the eigenfunctions $\xi_{\mathrm{r}}$ and the eigenfrequency $v$. They are computed with the adiabatic pulsation code FILOU (Tran Minh \& Leon 1995) for each model.

Finally, quantities which are related to the properties of the turbulent flow:

- the wavenumber $(k)$ dependency of $E$, i.e. the turbulent kinetic energy spectrum;

- the values and depth dependency of $k_{0}$, the wavenumber at which the convective energy has its maximum and is "injected" into the inertial range of the turbulent kinetic energy spectrum $E$;

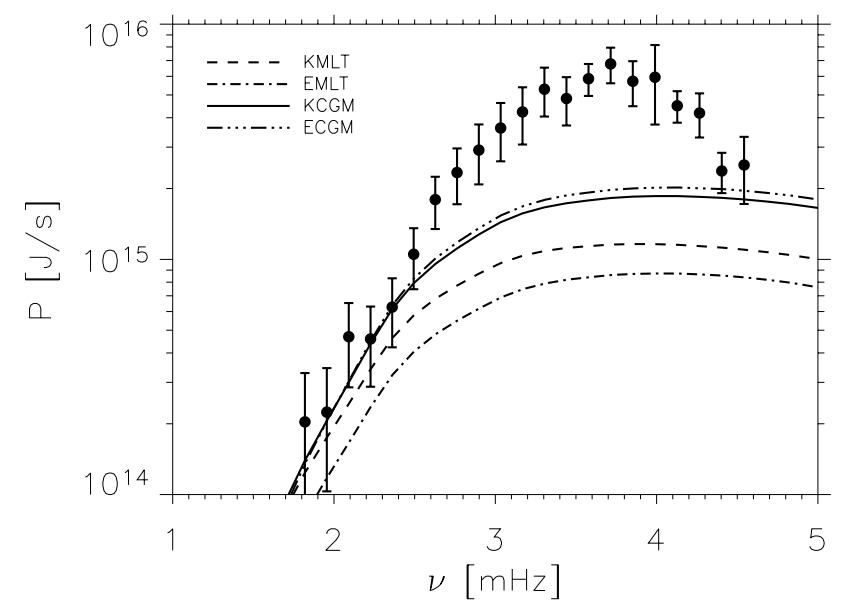

Fig. 4. The computed solar $p$ mode excitation rates, $P(v)$, are plotted versus the frequency for the KMLT (dashed line), EMLT (dot-dashed line), ECGM (dot-dot-dot-dashed line), and KCGM (solid line) models. The filled circles represent the "observed" solar values of $P(v)$ derived - according to Eq. (4) - from the amplitudes and line widths of the $\ell=0 p$-modes measured by Chaplin et al. (1998).

- the $v$-dependency of $\chi_{k}$, the frequency component of the auto-correlation product of the turbulent velocity field.

According to the results in Papers II and III obtained on the basis of a 3D numerical solar granulation simulation, the $k$-dependency of $E(k, v)$ is approximately reproduced by an analytical spectrum called "Extended Kolmogorov Spectrum" (EKS) and defined in Musielak et al. (1994). The $v$-dependency of $\chi_{k}$ is found to be better modelled with a Lorentzian function rather than by a Gaussian function, which is usually assumed for $\chi_{k}$ (see Paper III). Within most parts of the excitation region, the spatially averaged anisotropy factor $\bar{\Phi}$ is found almost constant with a value of $\bar{\Phi} \sim 2$ in agreement with BV's value.

At the top of the superadiabatic region, it was found that $k_{0} \sim 3.6 \mathrm{Mm}^{-1}$ and that $k_{0}$ decreases slowly inwards with depth (see Paper II). A good approximation for our region of interest within the Sun is to assume a constant $k_{0}$.

\subsection{Comparison with the observations}

Results for $P$ are presented in Fig. 4. The theoretical estimates for $P$ are compared with the "observed" $P_{\text {obs }}$, calculated from the seismic data of Chaplin et al. (1998) according to the relation:

$P_{\mathrm{obs}}(v)=2 \pi \Gamma \frac{I}{\xi_{\mathrm{r}}^{2}\left(r_{s}\right)} v_{s}^{2}(v)=2 \pi \Gamma \mathcal{M} v_{s}^{2}(v)$,

where: $v$ is the mode frequency; $r_{s}$ is the radius at which oscillations are measured;

$I \equiv \int_{0}^{M} \mathrm{~d} m \xi_{\mathrm{r}}^{2}$

is the mode inertia; $\mathcal{M}=I / \xi_{\mathrm{r}}^{2}\left(r_{s}\right)$ is the mode mass; and finally $\Gamma$ and $v_{s}$ are the mode line-width and the mode surface velocity, respectively, and are obtained from Chaplin et al. (1998). We point out that, according to the definition of Eq. (4), the derived value of $P_{\mathrm{obs}}$ depends on the model one considers through 


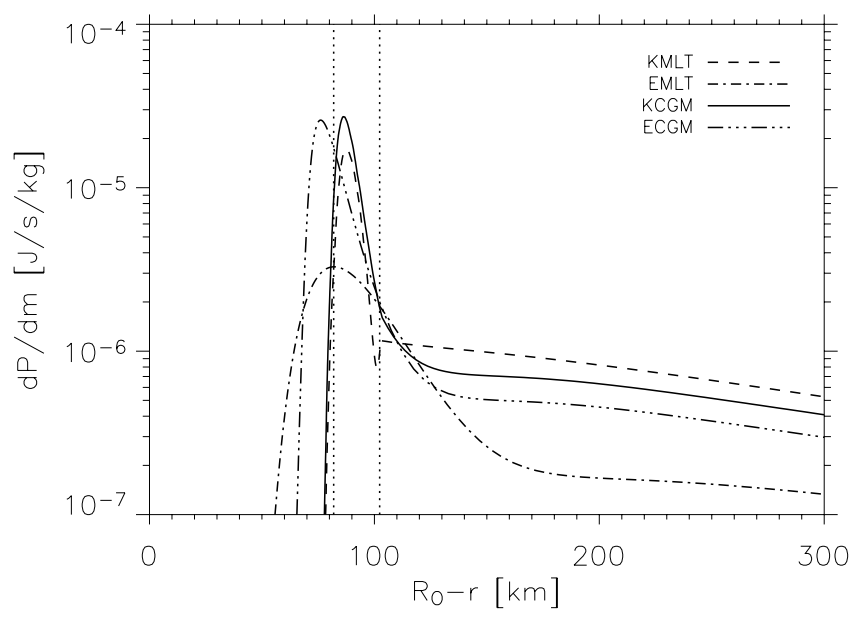

Fig. 5. The integrand $\mathrm{d} P / \mathrm{d} m$ (Eq. (2) of Paper II) is plotted versus $R_{\odot}-r$ for a mode with order $n=20$ ( $n=1$ being the fundamental mode) for the KMLT model (dashed line), EMLT model (dotdashed line), ECGM model (dot-dot-dot-dashed line), and KCGM model (solid line).

$\mathcal{M}$. Indeed, for a given mode, $\mathcal{M}$ - a priori - differs from one model to another. However, the mode masses of the models we consider here are very close to each other such that the changes on $P_{\text {obs }}$ due to the use of the mode mass of the different models are not significant compared to the error bars attached to the measurements. For each mode, we can then compute a unique value for $P_{\text {obs }}$ and compare the values of $P$ obtained for the different models to each other and to $P_{\text {obs }}$. We choose to derive $\mathcal{M}$ from the radial eigenfunctions $\xi_{\mathrm{r}}$ computed for the KCGM model and adopt $r_{s}=R_{\odot}+200 \mathrm{~km}$ consistently with the Chaplin et al. (1998) observations.

As shown in Fig. 4, differences between $P_{\text {KCGM }}$ and $P_{\text {ECGM }}$ are found smaller than the error bars associated with $P_{\text {obs }}$ from Chaplin et al. (1998). This is a consequence of the fact that the ECGM and KCGM models present very similar convective structures (see Sect. 3.3 and Fig. 3). On the other hand, $P_{\mathrm{KMLT}}$ is found significantly larger than $P_{\mathrm{EMLT}}$ as a consequence of the fact that the KMLT models result in larger $v$ and $F_{\mathrm{c}}$ values than the EMLT ones (see Sect. 3.3 and Fig. 3) for $\tau \gtrsim 10 \ldots 20$.

Furthermore, $P_{\mathrm{KCGM}}$ and $P_{\mathrm{ECGM}}$ are found closer to $P_{\mathrm{obs}}$ than $P_{\text {KMLT. }}$. However, above $v \gtrsim 2.5 \mathrm{mHz}$, differences between $P_{\text {obs }}$ and $P_{\mathrm{KCGM}}$ (or $P_{\mathrm{ECGM}}$ ) remain important. The origin of this discrepancy is discussed in Sect. 5.

For the KCGM and ECGM models, we have so far assumed $\bar{\Phi}=3$. According to Eq. (A.6), assuming $\bar{\Phi}=2-$ a value which is consistent with results from the $3 \mathrm{D}$ solar simulation - results in a driving by the entropy source smaller by a factor $\sim 2 / 3$ compared to the case $\bar{\Phi}=3$. This decrease, however, remains small compared to the difference in $P$ between the different models and hence does not influence our main results.

We present in Fig. 5 the integrand $\mathrm{d} P / \mathrm{d} m$ (Eq. (2) of Paper II) corresponding to the excitation rate $P$ of a mode of order $n=20$. The plot is done for the four solar models. For three of the four models the extent of the region where most of the excitation takes place is very thin (less than $50 \mathrm{~km}$ ). Obviously, this is the consequence of the very shallow extent of the superadiabatic region (see Fig. 2). This tiny extent of the excitation region strongly contrasts with the one found in Stein $\&$ Nordlund (2001) and in Paper III. For instance, those authors found a size of the order of $\sim 500 \mathrm{~km}$ for a mode of the same order. This discrepancy is attributed to the local nature of the convective treatments investigated in this work (see Sect. 5). Note that in Fig. 5 the EMLT model, with its large value of $\alpha$ for the entire superadiabatic region, predicts a broader excitation region than the other models. This is due to a smaller superadiabatic peak. However, in this model the transport of convective heat occurs with a smaller convective velocity and hence, as expected, the excitation is smaller than for the other models. We note here that despite the EMLT model results in a temperature structure closer to the numerical simulations (smaller superadiabatic peak), the predicted excitation amplitudes are smaller than for the other models investigated here and are the most discrepant in comparison with the data. This confirms that excitation rates provide a decisive additional test for convection models.

\section{Conclusions}

We have computed the rates $P$ at which acoustic energy is injected into the solar radial $p$ modes for several solar models. The solar models are computed with two different local treatments of convection: MLT and CGM.

For one set of solar models (EMLT and ECGM models), the atmosphere is gray and assumes Eddington's approximation. The models assume only one mixing-length parameter value and reproduce the solar radius at solar age but not the Balmer line profiles. For a second set of models (KMLT and KCGM models), the atmosphere is built using a $T(\tau)$ law which was obtained from a Kurucz's model atmosphere computed with the same local treatment of convection. The mixing-length parameter in the model atmosphere is chosen so as to provide good agreement between synthetic and observed Balmer line profiles, while the mixing-length parameter in the interior model is calibrated so that the model reproduces the solar radius at solar age.

Both the KMLT and the KCGM models reproduce the Balmer line profile and the solar radius and luminosity but - as shown in Figs. 2-4 - the CGM models model the transition between the region of high convective efficiency (the interior) and the region of low efficiency (the atmosphere) in a more realistic way than do the MLT models, as they reproduce the observed excitation rate $P$ more closely and predict a smoother transition region. Furthermore, the KMLT model requires a change of the mixing-length by a factor of five in a layer of $\sim 20 \mathrm{~km}$ thickness, which is significantly less than a pressure scale height $(\sim 300 \mathrm{~km})$. Given the meaning of alpha, this means that the mixing-length varies from about $150 \mathrm{~km}$ to $750 \mathrm{~km}$ in a layer of $\sim 20 \mathrm{~km}$ thickness (!), which does not make much sense from a physical point of view. On the other hand, the KCGM model does not require such large change in $\alpha$.

For the MLT treatment, the oscillation excitation rates, $P$, do significantly depend on the properties of the atmospheres investigated here. Indeed, differences in $P$ between the EMLT model and the KMLT model are found to be very large. On the other hand, for the CGM treatment, differences in $P$ between 
the ECGM and the KCGM models are very small compared to the error bars attached to the seismic measurements. This result shows that an Eddington gray atmosphere can be assumed for the calculation of $P$ when the CGM formulation is adopted. This would be particulary convenient in the case of massive computations of $P$ for a large set of stellar models.

For the EMLT and KMLT models, $P$ is significantly underestimated compared to the solar seismic constraints obtained from the Chaplin et al. (1998) measurements. KCGM and ECGM models yield values for $P$ closer to the seismic data than the EMLT and KMLT models. Contraints on the $\mathrm{H}_{\beta}$ Balmer line profile and on the solar radius can be satisfied by the two formulations (MLT and CGM), provided that the mixing-length parameters $\alpha_{\mathrm{i}}$ and $\alpha_{\mathrm{a}}$ are suitably adjusted. Once the above constraints are satisfied, the solar seismic data provide additional valuable constraints and, according to the present investigation (focussed on local approaches), they cleary favor the CGM treatment.

Our calculations are based here on Grevesse \& Noels (1993)'s solar abundances. There was recently a change in these values (see Asplund et al. 2004, 2005) with quite some implications for the internal structure of the Sun (see Basu \& Antia 2004; Montalbán et al. 2004; Bahcall et al. 2005; Antia $\&$ Basu 2005). Then the implication for the calculation of the excitation rates $(P)$ must in principle be tested consistently by changing the solar mixture both in the interior and the atmosphere (work in progress). However, we expect small changes in $P$. Indeed, as a first step we calculated a solar model having the low metallicity $Z / X=0.0171$ inferred from the new Asplund et al. (2004)'s revised solar abundances, in which the interior calculation is based on the detailed Asplund et al. mixture and associated opacities, while the atmosphere considers the low metallicity but keeps the Grevesse \& Noels (1993)'s solar abundances and associated opacities. Changes in $P$ smaller than $\sim 10 \%$ - much smaller than the errors bars $(\sim 20 \%)$ associated with the current measurements - were obtained.

The remaining discrepancy above $v \gtrsim 2.5 \mathrm{mHz}$ between computed and observed $P$ (Fig. 4 ) is attributed to the assumption of locality in the present treatment of convection. As a matter of fact, Samadi et al. (2003b, Paper III) have succeeded in reproducing the seismic constraints much better using constraints from a 3D solar granulation simulation. One reason for this improvement is that convection is intrinsically a nonlocal phenomenon. In the terminology of classical turbulence modelling, the eddies located at different layers contribute to the convective flux of a given layer (cf. also the discussion in Houdek 1996). Hence, a non-local description of convection is expected to predict a more extended superadiabatic region. This is suggested, for instance, by the comparison of our present results with that of Paper II. Non-local theories such as those by Gough (1977) and by Canuto \& Dubovikov (1998) - also support this explanation by typically predicting a smaller temperature gradient in the superadiabatic region than the local theories do (see Houdek 1996; Kupka \& Montgomery 2002), and thus a more extended superadiabatic region as well. Another property of solar granulation caused by non-locality is the observed asymmetry between the areas covered by upand downflows. This allows for a lower root mean square velocity while larger velocities (and thus more effective mode driving) can be reached in the downflows with their much higher velocities (note that such an asymmetry can be accounted for through non-local models as proposed by Canuto \& Dubovikov (1998, see also Kupka \& Montgomery 2002), although in a more simplified manner). On the other hand, the local models studied here cannot account for the different properties of up- and downflow areas at all. Hence, the superadiabatic region in these models is physically different from the one expected from non-local models and found in numerical simulations. Solar modes above $v \gtrsim 2 \mathrm{mHz}$, however, are predominantly excited in the superadiabatic region (Paper III). A larger amount of acoustic energy is then injected into those modes when convection is treated - as is in the $3 \mathrm{D}$ simulation or on the base of a non-local theory - in a more realistic manner than through local theories.

The results presented here so far only concern our Sun. Samadi et al. (2003a) found that $P$ scales as $(L / M)^{s}$ where $s$ is the slope of the power law and $L$ and $M$ are the mass and luminosity of their computed 1D stellar models. By building a set of stellar models with the MLT and another one with Gough's (1977) non-local formulation of convection, the authors found that the slope $s$ is quite sensitive to the 1D treatment of convection. In this respect, it would be interesting to compare the influence of the CGM formulation or of Canuto \& Dubovikov's (1998) non-local convection treatment on the value of $s$ and to test whether or not measurements of $P$ can - for stars other than the Sun - distinguish the best 1D treatment of convection.

Acknowledgements. R.S.'s work was supported in part by the Particle Physics and Astronomy Research Council of the UK under grant PPA/G/O/1998/00576 (who also supported FK), by the Société de Secours des Amis des Sciences (Paris, France) and by Fundacão para a Ciência e a Tecnologia (Portugal) under grant SFRH/BPD/11663/2002. We thank Achim Weiss for having carefully read this manuscript and R. Kurucz for giving access to the ATLAS9 code and associated data. Finally we thank the referee for his judicious suggestions.

\section{Appendix A: Relation between the entropy fluctuations and the convective flux}

As in Paper I, we relate the entropy fluctuations, $s_{t}$, to temperature fluctuations, $\theta$, as follows:

$s_{t}=\frac{c_{p}}{T_{0}} \theta$

where $T_{0}$ is the mean temperature and $c_{p}=(\partial s / \partial \ln T)_{p}$. Hence, the mean square of the entropy fluctuations, $\left\langle s_{t}^{2}\right\rangle$, can be expressed as

$\left\langle s_{t}^{2}\right\rangle \approx\left(\frac{c_{p}}{T_{0}}\right)^{2}\left\langle\theta^{2}\right\rangle$.

The convective flux is related to $\theta$ and $u_{z}$, the vertical component of convective velocity, as

$F_{\mathrm{c}} \approx \rho_{0} c_{p}\left\langle u_{z} \theta\right\rangle$ 
where $\rho_{0}$ is the mean density. We furthermore assume, consistently with the adopted quasi-normal approximation in Paper I, that $\left\langle u_{z} s_{t}\right\rangle^{2}$ can be decomposed as

$\left\langle u_{z} s_{t}\right\rangle^{2}=\left\langle u_{z}^{2}\right\rangle\left\langle s_{t}^{2}\right\rangle=w^{2}\left\langle s_{t}^{2}\right\rangle$.

Finally, one can show that

$\alpha_{s} \equiv\left(\frac{\partial p}{\partial s}\right)_{\rho}=\rho_{0} T_{0} \Gamma_{1} \nabla_{\mathrm{ad}}$,

where $s$ is the entropy and $p$ the pressure, $T_{0}$ is the mean temperature, $\Gamma_{1}=(\partial \ln p / \partial \ln \rho)_{s}$ is the adiabatic exponent, and $\nabla_{\mathrm{ad}}=(\partial \ln T / \partial \ln p)_{s}$ is the adiabatic temperature gradient.

The mean square of the entropy fluctuations can then be deduced from the set of Eqs. (A.1)-(A.5) and (2):

$\left\langle s_{t}^{2}\right\rangle \approx \frac{\bar{\Phi}}{3}\left(\frac{F_{\mathrm{c}}}{\rho_{0} T_{0} u_{0}}\right)^{2}$,

where $\bar{\Phi}$ is the spatially averaged anisotropy factor, which is defined in Eq. (2). Driving by the entropy source term is then proportional to $\left(\alpha_{s} / \rho_{0} T_{0}\right)^{2}(\bar{\Phi} / 3) F_{\mathrm{c}}^{2}-$ see Paper I - and thus scales like the square of $F_{\mathrm{c}}$.

\section{References}

Antia, H. M., \& Basu, S. 2005, ApJ, 620L, 129

Alexander, D. R., \& Ferguson, J. W. 1994, ApJ, 437, 879

Asplund, M., Grevesse, N., Sauval, A. J., et al. 2004, A\&A, 417, 751

Asplund, M., Grevesse, N., \& Sauval, A. J. 2005, in Cosmic Abundances as Records of Stellar Evolution and Nucleosynthesis, ASP Conf. Ser., 336, 25 [arXiv: astro-ph/0410214]

Bahcall, J. N., Basu, S., Pinsonneault, M., \& Serenelli, A. M. 2005, ApJ, 618, 1049

Balmforth, N. J. 1992, MNRAS, 255, 639

Basu, S. 1997, MNRAS, 288, 572

Basu, S., \& Antia, H. M. 2004, ApJ, 606L, 85

Bernkopf, J. 1998, A\&A, 332, 127

Böhm-Vitense, E. 1958, Zeitschr. Astrophys., 46, 108

Canuto, V. M. 1992, ApJ, 392, 218

Canuto, V. M. 1993, ApJ, 416, 331

Canuto, V. M., \& Mazzitelli, I. 1991, ApJ, 370, 295 (CM)

Canuto, V. M., \& Dubovikov, M. 1998, ApJ, 493, 834

Canuto, V. M., Goldman, I., \& Mazzitelli, I. 1996, ApJ, 473, 550 (CGM)

Caughlan, G. R., \& Fowler, W. A. 1988, Atomic Data and Nuclear Data Tables, 40, 283

Chaplin, W. J., Elsworth, Y., Isaak, G. R., et al. 1998, MNRAS, 298, L7
Christensen-Dalsgaard, J., \& Daeppen, W. 1992, A\&AR, 4, 267

Christensen-Dalsgaard, J., Gough, D. O., \& Thompson, M. J. 1991, ApJ, 378, 413

Cox, J. 1968, Principles of stellar structure (Gordon and Breach)

Fuhrmann, K., Axer, M., \& Gehren, T. 1993, A\&A, 271, 451

Fuhrmann, K., Axer, M., \& Gehren, T. 1994, A\&A, 285, 585

Goldreich, P., \& Keeley, D. A. 1977, ApJ, 212, 243

Gough, D. O. 1977, ApJ, 214, 196

Grevesse, N., \& Noels, A. 1993, in Origin and Evolution of the Elements, ed. N. Prantzos, E. Vangioni-Flam, \& M. Cassé (Cambridge University Press), 15

Guenther, D. B., Demarque, P., Kim, Y.-C., \& Pinsonneault, M. H. 1992, ApJ, 387, 372

Heiter, U., Kupka, F., van't Veer-Menneret, C., et al. 2002, A\&A, 392, 619

Houdek, G. 1996, Ph.D. Thesis, Institut für Astronomie, Wien

Houdek, G., Balmforth, N. J., Christensen-Dalsgaard, J., \& Gough, D. O. 1999, A\&A, 351, 582

Iglesias, C. A., \& Rogers, F. J. 1996, ApJ, 464, 943

Kupka, F., \& Montgomery, M. H. 2002, MNRAS, 330, L6

Kurucz, R. L. 1992, Rev. Mex. Astron. Astrofis., 23, 45

Kurucz, R. L. 1993, CD-ROM 13, Cambridge, SAO

Michaud, G., \& Proffitt, C. R. 1993, in IAU Colloq., 137, Inside the Stars, ASP Conf. Ser., 40, 246

Montalbán, J., D’ Antona, F., Kupka, F., \& Heiter, U. 2004, A\&A, 416, 1081

Montalbán, J., Miglio, A., Noels, A., Grevesse, N., \& di Mauro, M. P. 2004, in Proc. SOHO, 14, Helio- and Asteroseismology: Towards a Golden Future, ESA SP-559, 574

Morel, P. 1997, A\&AS, 124, 597

Morel, P., van't Veer, C., Provost, J., et al. 1994, A\&A, 286, 91

Musielak, Z. E., Rosner, R., Stein, R. F., \& Ulmschneider, P. 1994, ApJ, 423, 474

Rosenthal, C. S., Christensen-Dalsgaard, J., Nordlund, Å., Stein, R. F., \& Trampedach, R. 1999, A\&A, 351, 689

Samadi, R., \& Goupil, M. J. 2001, A\&A, 370, 136 (Paper I)

Samadi, R., Goupil, M. J., Lebreton, Y., Nordlund, A., \& Baudin, F. 2003a, Ap\&SS, 284, 221

Samadi, R., Nordlund, A., Stein, R. F., Goupil, M. J., \& Roxburgh, I. 2003b, A\&A, 404, 1129 (Paper III)

Samadi, R., Nordlund, ̊̊., Stein, R. F., Goupil, M. J., \& Roxburgh, I. 2003c, A\&A, 403, 303 (Paper II)

Samadi, R., Georgobiani, D., Trampedach, R., et al. 2005, A\&A, submitted

Schlattl, H., Weiss, A., \& Ludwig, H.-G. 1997, A\&A, 322, 646

Smalley, B., \& Kupka, F. 1997, A\&A, 328, 349

Stein, R. F., \& Nordlund, Å. 2001, ApJ, 546, 585

Tran Minh, F., \& Leon, L. 1995, in Physical Process in Astrophysics, 219

van't Veer-Menneret, C., \& Megessier, C. 1996, A\&A, 309, 879

Xiong, D. R. 1978, Chin. Astron., 2, 118

Xiong, D. R. 1985, A\&A, 150, 133 